\title{
e-Migrinter
}

$11 \mid 2013$

Et l'immobilité dans la circulation?

\section{Synthèse des débats}

Céline Bergeon, Françoise Dureau, Christophe Imbert, Guillaume Le Roux et David Lessault

\section{OpenEdition}

\section{Journals}

Édition électronique

URL : https://journals.openedition.org/e-migrinter/272

DOI : $10.4000 /$ e-migrinter.272

ISSN : 1961-9685

Éditeur

UMR 7301 - Migrinter

Édition imprimée

Date de publication : 1 septembre 2013

Pagination : 184-187

ISSN : 1961-9685

\section{Référence électronique}

Céline Bergeon, Françoise Dureau, Christophe Imbert, Guillaume Le Roux et David Lessault,

«Synthèse des débats », e-Migrinter [En ligne], 11 | 2013, mis en ligne le, consulté le 20 mai 2021.

URL : http://journals.openedition.org/e-migrinter/272 ; DOI : https://doi.org/10.4000/e-migrinter.272 


\section{Synthèse des débats}

Céline Bergeon, Françoise Dureau, Christophe Imbert, Guillaume Le Roux \& David Lessault

A

u fil de ces deux journées, deux réflexions transversales ont plus spécifiquement accompagné les échanges et nourri les débats autour des réflexions développées dans des contextes variés par chacun des intervenants.

La première concerne le caractère subi/choisi de la circulation, le degré de contrainte plus ou moins fort qui pèse sur les pratiques sociales et spatiales, qui est loin de concerner uniquement la session dédiée à l'immobilité forcée. La seconde renvoie à la question des échelles spatio-temporelles et de leur articulation : d'ordre technique de prime abord, elle nous confronte plus fondamentalement au débat incontournable des catégories utilisées pour qualifier et rendre compte des comportements individuels et collectifs.

\section{L'immobilité : entre contrainte et stratégie}

La question du choix est transversale à l'ensemble des présentations. Elle apparaît en premier lieu sous l'angle d'une privation de l'autonomie plutôt que de celui de la privation de la mobilité, comme l'ont bien souligné Bénédicte Michalon, Lucie Bony, Fleur Guy, Nathalie Ortar et Sandrine Depeau dans leurs interventions. En effet, elles ont signalé tour à tour l'importance du contrôle du contact spatial et social - des détenus, des adolescents placés en centre, des femmes et des enfants - qui s'opère à partir de pratiques de clôture, de fermeture, «d'orientation» de la mobilité ou encore d'assignation sociale (Nathalie Ortar). Les acteurs de l'encadrement font office "d'opérateurs de la mobilité et de l'immobilité » (Sandrine Depeau), définis aussi comme des «régulateurs» par Fleur Guy. Le vocabulaire utilisé par les intervenants, pour évoquer cette privation de l'autonomie dans la mobilité, alimente le caractère contraint (" mobilité subie et encadrée », cf. Bénédicte Michalon) et orienté ( "mobilité canalisée », " trajet ambulé », cf. Sandrine Depeau) de l'immobilité et de la mobilité.

En second lieu, la notion de choix se manifeste également sous l'angle de la «stratégie». Les contributions de Laure Carbonnel et de Thomas Pfirsch ont notamment montré le rôle de l'immobilité dans la préservation de pratiques sociales et cultuelles ainsi que dans la reproduction sociale du groupe et/ou de la famille. Ces communications nous ont permis 
d'envisager l'immobilité comme une stratégie et d'attribuer à celle-ci un rôle déterminant dans les pratiques de ceux qui sont mobiles. Le couple immobilité/mobilité a pris sens dans cette unité et non en tant que pratiques sociales et spatiales indépendantes l'une de l'autre. L'alternance des positions des individus dans la mobilité, puis dans l'immobilité, a vraiment révélé cette interdépendance des pratiques qui s'entretiennent continuellement, comme l'a souligné Christophe Imbert lors du débat de clôture. Ce dernier a insisté, à juste titre, sur le rôle moteur de l'ancrage dans la reproduction (et la maitrise) de la mobilité et sur la nécessité de dépasser le caractère négatif souvent assigné à l'immobilité. Le mobile ne circule qu'en référence à des lieux fixes qui ne prennent sens que dans un système de lieux où chaque individu régule ses déplacements, ses références, ses pratiques, etc. Ce système de lieux ferait l'objet de (re)négociations, de ruptures, ou encore de continuités de la part des individus, et permettrait, d'un point de vue heuristique, de lever l'ambiguité tournant autour de la dichotomie mobilité/immobilité (Françoise Dureau). La mobilité ne prendrait donc son véritable sens qu'à travers des moments d'immobilité, et vice versa; laissant place à des «intermittences saisonnières » et à des «sédentarités multilocalisées » comme l'a expliqué Cédric Duchêne-Lacroix à propos de la relation entre la dispersion géographique des lieux de vie et d'habitation, et le développement des rythmes de la mobilité. Ce lien étroit entre mobilité et immobilité a également été souligné par Evelyne Mesclier dans le cas des paysanneries péruviennes: la mobilité de certains membres de la famille permettrait le maintien d'un accès à la terre pour d'autres.

La question du choix a souvent été associée dans plusieurs présentations à celle des ressources. Les contributions de Lucie
Bony et de Thomas Pfirsch ont clairement explicité les moyens déployés par les individus pour dépasser l'assignation sociale et spatiale. Si l'immobilité est une stratégie, sa concrétisation fait appel à des ressources de natures économique, sociale, familiale, etc. L'exemple des détenus (Lucie Bony) montre comment les individus peuvent s'approprier l'immobilité «forcée» pour la dépasser ou bien la reconfigurer puisque celle-ci est considérée comme une donne qui fait partie du quotidien. La circulation d'informations, d'objets ou encore de «consignes» entre le détenu et ses relations extérieures, montre comment celui-ci est aussi présent à distance et s'inscrit clairement dans une ubiquité résidentielle qui lui permet de maitriser une immobilité imposée. Dans ce cas d'immobilité contrainte, l'ubiquité peut se matérialiser par une «délégation de la mobilité » entre plusieurs individus (Françoise Dureau). Dans d'autres présentations, cette question des ressources a été abordée dans le cadre d'arbitrages opérés par les individus pour tempérer le poids de la contrainte d'une vie mobile, ou encore pour trouver un nouvel équilibre dans le quotidien: Nathalie Ortar a particulièrement bien montré comment, au sein du couple, les femmes essaient de négocier, malgré le poids de l'assignation sociale, leur rôle dans la structure familiale au moment d'une mobilité professionnelle.

\section{L'espace-temps de l'immobilité et de la mobilité}

La question de la temporalité, présente dans l'ensemble des contributions, montre que les pratiques sont autant conditionnées par l'espace que par le temps. Ce constat est exacerbé dans les situations d'immobilité subie et régulée qui nous ont été décrites. Bénédicte Michalon a expliqué comment les divisions du temps (des moments de la 
journée) influençaient les divisons de l'espace et engendraient ainsi une segmentation spatiale et temporelle des pratiques des détenus dans les prisons roumaines. Ce contrôle du temps influence en effet les moments de mobilité et d'immobilité et inscrivent les individus dans des pratiques normalisées. C'est également ce qu'observent Fleur Guy et Sandrine Depeau lorsqu'elles évoquent le rapport entre immobilité et pratiques réticulaires des lieux sous le contrôle duquel se déroule la mobilité quotidienne.

À une autre échelle, Cédric DuchêneLacroix et Thomas Pfirsch ont aussi insisté sur la relation entre pratiques de mobilité/immobilité et différentes phases de la vie. L'imbrication des échelles du temps et de l'espace (que celles-ci soient définies en fonction des grands événements de la vie ou en fonction du quotidien) montre encore une fois que les individus alternent sur le temps long, périodes de mobilité et périodes de sédentarité selon des rythmes sans cesse redéfinis. La question des temporalités et des échelles du temps a donc été un axe transversal à l'ensemble des communications de ces deux journées tant du point de vue de la reproduction et/ou du contournement des modèles culturels (Laure Carbonnel), familiaux et économiques (Thomas Pfirsch, Nathalie Ortar, Evelyne Mesclier) que du point de vue méthodologique (Guillaume Le Roux). Les difficultés méthodologiques liées à l'articulation (parfois à la superposition) des pratiques spatiales renforcent l'idée principale selon laquelle les comportements en termes de déplacements et de fixité sont difficilement dissociables.

À travers l'ensemble des communications, nous avons vu que la mobilité et l'immobilité se transforment en fonction des règles spatiales, sociales et temporelles et que différentes normes collectives conditionnent et font évoluer les pratiques. Parfois, ces règles cloisonnent l'immobilité et la mobilité (comme dans le cas des détenus et des enfants, par exemple), alors que, dans d'autres cas, elles en complexifient les contours. $\grave{A}$ plusieurs reprises, le poids de l'immobilité dans la reproduction de la mobilité a été évoqué : l'immobilité permet les rencontres et se pose en moment de (re)négociation de la mobilité. La période de fixité permet, entre autres, d'entretenir et de renforcer pour chacun, le capital de mobilité. Aussi, les arrangements et les adaptations qui ont été évoqués tout au long des présentations, montrent-ils clairement l'existence d'un savoir-circuler mais également d'un savoir-faire dans l’immobilité (Bénédicte Michalon).

Enfin, cette vision dynamique du couple mobilité/immobilité et notamment du sens donné à la fixité et ses modalités de mise en œuvre, nous a conduits à nous interroger sur un vocabulaire plus adéquat pour décrire ces situations imbriquées et jamais définitives. Au terme de cette journée d'étude, l'utilisation du terme «d'immobilisation» plutôt que de celui «d'immobilité » est apparue plus pertinente dans la description et l'analyse de ces situations et de ces pratiques, montrant toute la complexité du processus qui anime la mobilité et l'immobilité. Cette vision permet de ne pas trop enfermer mobiles et immobiles dans certaines catégories préconçues. Le souci de décloisonnement des catégories a ainsi alimenté en continu le propos des présentations et a révélé l'importance qu'il y a à considérer avec prudence ces assignations. Comme l'a mentionné Evelyne Mesclier, la tendance à considérer trop rapidement l'objet « mobilité » est aujourd'hui très prégnante, ne serait-ce que par le sens attribué au déplacement. Les débats auxquels nous avons contribué montrent bien la nécessité de considérer les pratiques de mobilité et 
d'immobilité comme des décisions négociées et de donner une place essentielle à la notion d'ubiquité, en tant que capacité des individus à assurer une présence continue dans plusieurs lieux; ouvrant ainsi de nouvelles perspectives d'analyse du point de vue des échelles du temps et de l'espace.

Céline Bergeon

ATER Université de Limoges, UMR 7301

Migrinter bergeonceline@yahoo.fr

Françoise Dureau

DR IRD honoraire, UMR 7301 Migrinter

f.dureau@gmail.com
Christophe Imbert Maître de conférences, Université de Poitiers, UMR 7301 Migrinter Accueilli en délégation à l'INED - UR 06

« Mobilité, Logement et Entourage » christophe.imbert@univ-poitiers.fr

Guillaume Le Roux Doctorant Université de Poitiers, UMR 7301

Migrinter guillaumeleroux14@gmail.com

David Lessault CR CNRS, UMR 7301 Migrinter Associé à l'INED - UR 06 « Mobilité, logement et entourage » david.lessault@univ-poitiers.fr 